\title{
École et migration en Guyane française : invisibilité des parcours migratoires dans les dossiers scolaires à Maripasoula
}

School and Migration in French Guiana: Invisibility of Migratory Routes in the Scholastic Folders in Maripasoula

Escuela y migración en la Guayana francesa: invisibilidad de las rutas migratorias en los registros escolares en Maripasoula

\section{Alexandra Vié}

\section{(2) OpenEdition}

\section{Journals}

Édition électronique

URL : https://journals.openedition.org/remi/11811

DOI : 10.4000/remi. 11811

ISSN : $1777-5418$

Éditeur

Université de Poitiers

Édition imprimée

Date de publication : 1 décembre 2018

Pagination : 143-165

ISBN : 979-10-90426-63-4

ISSN : 0765-0752

Référence électronique

Alexandra Vié, «École et migration en Guyane française : invisibilité des parcours migratoires dans les dossiers scolaires à Maripasoula », Revue européenne des migrations internationales [En ligne], vol. 34 n4 | 2018, mis en ligne le 01 janvier 2021, consulté le 14 avril 2022. URL : http://

journals.openedition.org/remi/11811; DOI : https://doi.org/10.4000/remi.11811 


\title{
École et migration en Guyane française : invisibilité des parcours migratoires dans les dossiers scolaires à Maripasoula
}

\section{Alexandra Vié ${ }^{1}$}

\begin{abstract}
"Lucas² est un jeune garçon né en 2007 au Suriname. À la lecture de son dossier scolaire, nous constatons qu'il possède un passeport brésilien. Il serait arrivé à l'école primaire de Maripasoula, en classe de CE2 en avril 2016. Dans son dossier, une adresse indiquait un domicile dans le bourg de la commune. À la rentrée 2017, Lucas est absent de l'école alors qu'il est inscrit en CM1. II arrive en décembre et une nouvelle adresse est donnée. Sans que cette dernière soit changée, en avril 2018 il déménage du côté du Suriname et termine son année scolaire à l'école. " (Notes de terrain, 2018)
\end{abstract}

Cet extrait de notes de terrain a été construit à l'aide de différents documents retrouvés dans le dossier scolaire de Lucas. S'intéresser aux papiers, qu'ils soient document d'identité, justificatif de domicile ou autre, c'est considérer qu'ils remplissent un rôle, une fonction. Pour Dardy (1997 : 225), "ces papiers multiples disent que nous participons d'un système généralisé d'inscriptions, assignant des places écrites et définissant des identités sociales". La place assignée par les papiers dépend en partie de la nature de ces derniers. L'identité scolaire de l'élève, à travers sa carte scolaire ou sa carte de cantine n'est que temporaire et localisée, contrairement à l'acte de naissance ou la carte nationale d'identité qui renvoient à une appartenance plus large, celle d'un État. Ces derniers font partie de l'état civil d'une personne. D'après la Cour de cassation, I'acte d'état civil est " un écrit dans lequel l'autorité publique constate d'une manière authentique, un évènement dont dépend l'état d'une ou de plusieurs personnes ${ }^{3}$ ". II s'agit d'une procédure écrite d'identification, constatant certaines caractéristiques d'un individu telles que sa naissance, ses liens de filiation ou encore son décès. Cependant, l'acte d'état civil n'est pas uniquement une finalité, qui se traduirait en une existence administrative, il est aussi "la preuve authentique de la situation juridique du sujet de droit " (Neirinck, 2008 : 17). II est la reconnaissance de l'individu en tant que sujet de droit et donc un

1 Doctorante, sciences de l'éducation, GRHAPES, INS-HEA, 58-60 avenue des Landes, 92150 Suresnes; alexandramarie.vie@gmail.com

2 Afin de respecter l'anonymat des enquêtés, tous les prénoms présents dans cet article ont été modifiés.

3 Cour de Cassation, chambre civile 1, du 14/06/1983, n 82-247. 
moyen nécessaire pour accéder aux droits liés à cette existence. Comme le dit Farge (2003: 45), "être identifié, appartenir à un système légitime et en donner la preuve par les papiers que l'on porte, c'est marquer que le corps est saisi par le droit». Que se passe-t-il quand l'identité papier n'existe pas ou encore quand elle ne peut être prouvée par des papiers ? Pour plusieurs auteures telles que Dardy (1997 et 1998), Benoît (2015 et 2016) ou Jamoulle (2013), I'existence papier est indispensable. "Qui n'est pas inscrit n'est pas seulement "marginal" catégorie propre à la culture écrite, qui définit des marges -, mais se retrouve plus radicalement privé d'existence " (Dardy, 1997 : 226). Sans papiers d'identité, il est difficile d'accéder au droit. Par exemple, il est impossible d'inscrire un enfant à l'école sans que ce dernier ait un acte de naissance ou une preuve de son identité ou de sa filiation.

Puisque l'accès à la scolarité semble être conditionné par une série de papiers, nous souhaitons, par ces documents, appréhender les trajectoires familiales d'enfants et d'adolescents migrants en Guyane française. Dans quelle mesure, est-il possible de reconstituer le parcours des enfants par des traces papier ? Quels éléments du parcours migratoire et familial sont mis en avant et quels sont ceux passés sous silence ? Voici un ensemble de questions qui soustendent notre réflexion. À partir des traces écrites laissées par les familles et les professionnels de l'éducation dans les dossiers scolaires, nous tenterons de mettre en mots des parcours de vie. Dans cet article, il s'agit moins d'analyser la production des catégories proposées par l'institution scolaire que de tenter de reconstruire des parcours à partir des traces papier. Nous partons du postulat que certains éléments sont mis en avant dans les dossiers tandis que d'autres sont passés sous silence, donnant ainsi au professionnel qui les consulte une vision tronquée des mobilités et déplacements des enfants et des adolescents. Pour y parvenir, nous avons choisi de travailler dans la plus grande commune de France, située en Guyane française, à la frontalière du Suriname et du Brésil : Maripasoula.

Pour ce faire, nous nous appuyons sur une enquête de terrain qualitative réalisée dans deux établissements scolaires du bourg de la commune : une école primaire et un collège. Cet article s'inscrivant dans un projet de recherche ${ }^{4}$ sur l'accueil et l'inclusion scolaire des élèves allophones nouvellement arrivés, nous avons choisi ces établissements, seuls de la commune à être pourvus $d^{\prime}$ 'une UPE2A ${ }^{5}$. Afin d'interroger les parcours, nous avons décidé de diversifier les méthodes de recueil de données. Nous avons allié des méthodes classiques

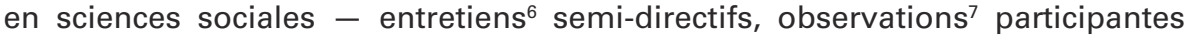

\footnotetext{
4 Projet de recherche : "Accueillir, sçolariser et accompagner les élèves primo arrivants, itinérants ou de famille du voyage. Élèves et professionnels du champ éducatif face aux enjeux de I'inclusion" ; EDUCINCLU, INSHEA, Suresnes. Ce projet comprend des terrains de recherche dans trois académies dont la Guyane.

5 UPE2A : Unité Pédagogique pour Élèves Allophones Arrivants, créée par la circulaire du ministère de l'Éducation nationale $n^{\circ}$ 2012-141 du 02/10/2012 intitulée "Organisation de la
} scolarité des élèves allophones nouvellement arrivés".

6 Une vingtaine d'entretiens semi-directifs ont été réalisés avec des professionnels de l'éducation : équipe de direction, personnel enseignant, personnel de santé ainsi qu'avec des acteurs du secteur médical (médecins, sage-femme) et associatif.

7 Des observations ont été menées dans les établissements scolaires, dans la cour de récréation, dans les salles de cours, lors de réunions entre équipes. 
et non participantes dans les classes - à des méthodes participatives ${ }^{8}$ avec les enfants. Dans la lignée des travaux de Béliard et Biland (2008: 106), il a été choisi d'étudier les dossiers scolaires comme un outil d'analyse supplémentaire pour multiplier les points de vue sur notre objet. Nous avons ciblé plusieurs éléments dans les dossiers : I'état civil avec notamment le lieu de naissance, la situation familiale, l'adresse, le parcours scolaire antérieur ainsi que toute trace ou mention d'un déplacement ou d'une migration. Nous nous sommes intéressées également à tous les justificatifs présents. Menant cette recherche depuis octobre 2016, il a été possible d'accéder à ces données. En effet, pour Béliard et Biland (2008 : 107-108) ainsi que Cayouette-Remblière (2011 : 119), la consultation des dossiers ne peut se faire qu'après avoir réalisé un terrain régulier et long.

Dans la première partie de l'article, nous reviendrons sur l'accès aux droits en Guyane et plus précisément sur le fleuve Maroni et nous insisterons sur l'invisibilité juridique de certaines populations. Dans une deuxième partie, nous présenterons les enjeux contemporains de scolarisation dans l'Ouest guyanais et les obstacles que doivent surmonter les familles pour inscrire leur enfant à l'école. Enfin, dans une troisième partie, nous ferons parler les papiers. Nous retracerons le parcours de vie et de mobilité des familles en confrontant les informations contenues dans les dossiers et l'expérience racontée par ces familles. Ce récit à deux voix a pour objectif de mettre en mots les parcours souvent passés sous silence.

\section{Exister sur le fleuve Maroni : une lente reconnaissance des populations}

\section{Une invisibilité juridique de la colonisation à la francisation}

"Les papiers sont à la fois ceux de la contrainte, du contrôle et même du contrôle d'État, mais ils sont aussi pourvoyeurs d'identité. L'identité — du moins une certaine forme d'identité - que chacun d'entre nous se joue et se rejoue se dessine, s'estompe ou s'affirme par ces papiers. " (Dardy, $1998:$ 13)

En France, c'est l'organisation étatique et plus souvent communale, qui est chargée de collecter, enregistrer et classer les informations relatives aux personnes. En effet, cette existence ne peut être officialisée que si elle est reconnue par l'autorité publique. Avant la Révolution française, les registres paroissiaux tenus par l'Église catholique constituaient l'état civil. Ainsi, seuls les catholiques en bénéficiaient. Le processus de sécularisation de l'état civil achevé par la loi du 20 septembre 1792 généralisa l'état civil comme mode de preuve de l'état des personnes et devint accessible à tout individu, quels que soient sa religion et son statut. Selon Noiriel (1993:3), cette décision était une conséquence directe de la Constitution du 3 septembre 1791.

"Le pouvoir législatif établira pour tous les habitants, sans distinction, le mode par lequel les naissances, les mariages et les décès seront constatés ; et il désignera les officiers publics qui en recevront et conserveront les actes. " (Titre II, art. 7)

8 Réalisation d'ateliers en UPE2A permettant aux enfants de s'exprimer sur leur ressenti scolaire. 
Inscrites dans I'histoire de France, nous pouvons penser, à l'instar de Noiriel (1993 : 27), que les formalités d'état civil sont aujourd'hui une «routine administrative", pour les personnes qui les engagent. Cependant, ces démarches ne semblent pas être vécues comme telle sur tous les territoires de France - anciens territoires coloniaux notamment - ni même pour certaines catégories d'acteurs, les étrangers par exemple. En effet, en Guyane française, l'état civil fut, jusqu'à très récemment, loin d'être une routine, en particulier sur les fleuves-frontière Maroni et Oyapock. Historiquement, vivent sur le fleuve Maroni, des peuples Marrons et Amérindiens. Les Marrons ${ }^{9}$ ou Noirs-Marrons également appelés "Bushinengues" (hommes de la forêt) sont pour la majorité des descendants d'esclaves déportés d'Afrique vers le Suriname, ancienne colonie hollandaise, qui ont fui les exploitations pendant le temps de l'esclavage. Ils sont nés des grands mouvements de marronnage (Doumeng, 2008). Ces deux peuples sont à distinguer des populations issues de l'esclavage qui entrèrent dans le droit commun après l'abolition de l'esclavage en 1848. Alors que les Amérindiens furent considérés comme des peuples autochtones, les peuples Marrons ont vu leur situation étroitement imbriquée à la définition juridique de la frontière fluviale franco-néerlandaise : le Maroni (Urban, 2015 : 427-428). Des traités de paix ont été établis entre la France, le Pays-Bas et les Marrons dès le milieu du XIXe siècle ${ }^{10}$. Cependant, les Marrons n'obtinrent aucune reconnaissance juridique et donc aucun état civil. Par exemple, parmi les Marrons, les Ndujka étaient considérés comme les alliés des autorités coloniales hollandaises. Par les différents traités de paix, la France entérina le principe de domination du gouvernement de la Guyane hollandaise sur les Ndujka et leur reconnut le droit de libre circulation sur le fleuve Maroni.

Dans les années 1930, la France réfléchit à implanter les premiers postes administratifs à l'intérieur du territoire et sur les fleuves. Thabouillot (2015: 438) rappelle que "I'arrière-pays de la Guyane abritait vers 1930 une société faite de groupes aux trajectoires historiques variés (Amérindiens, Marrons, migrants de I'or)". Le décret du 6 juin 1930 créa ainsi le territoire autonome de I'Inini. II s'agissait d'une unité administrative gérée directement par l'État avec à sa tête un gouverneur qui distinguait les populations nationales (créoles et françaises), des peuples indépendants (les Marrons et les Amérindiens).

"Le territoire autonome de l'Inini est sous administration directe de l'État, d'abord sous l'autorité du gouverneur, ensuite, avec la départementalisation, sous celle du préfet, représenté sur place par la gendarmerie. Ce découpage du territoire entérine la distinction démographique et politique entre, d'une part, les populations créole et métropolitaine dont les membres sont des nationaux et des citoyens français et, d'autre part, celles des Noirs marrons et des Amérindiens qualifiées de primitives et de tribales ; "peuples indépendants" dont les membres, pour la très grande majorité, n'ont ni état civil ni, en conséquence, nationalité et citoyenneté française. " (Benoît, $2016: 241$ )

Le gouvernement de I'Inini développa en 1936 une politique en lien avec les orientations générales du Front populaire et sous l'impulsion de la "doctrine

9 II existe différentes ethnies en Guyane : les Saramaca, les Ndjuka, les Boni ou Aluku, les Parmaca. À Maripasoula, I'ethnie Boni ou Aluku est majoritaire.

10 La Convention franco-néerlandaise du 08 novembre 1836, la Convention franco-ndjuka du 11 septembre 1860. 
indigène" (Thabouillot, $2015:$ 441). Après la départementalisation de 1946, le territoire de I'Inini devint I'arrondissement ${ }^{11}$ de I'Inini et conserva son statut particulier, permettant aux "peuples indépendants" de vivre conformément au droit coutumier, en marge des systèmes juridiques et assimilationnistes en place. Contre la volonté des élus guyanais, l'État décida de maintenir ce régime. Cependant, le courant assimilationniste revendiqué par les élus locaux et le contexte de décolonisation engagé par la France supprima ce régime par le décret du 17 mars 1969. Le système des communes fut alors instauré, créant, entre autres la commune de Maripasoula. En 1962, "57 \% de la population de I'Inini était d'origine "tribale" et "les non tribaux" étaient à $23 \%$ des Français et à $20 \%$ des étrangers non tribaux" (Thabouillot, $2015: 446$ ). Dès lors, il fallait disposer d'un nombre de citoyens français suffisants pour permettre l'exercice de la vie politique municipale de ces nouvelles communes. C'est ainsi que l'administration accéléra le recensement des populations pour permettre d'établir un état civil et la nationalité des populations tribales dès 1956.

"La première campagne d'établissement d'un état civil, via les JDN [jugements déclaratifs de naissance], condition nécessaire d'attribution de la nationalité, remonte à 1956 et fait suite à des instructions préfectorales datant de la même année ; la deuxième est initiée en 1965, à la demande du ministère de la Justice. " (Benoît, 2016 : 242)

Parmi les Marrons, les Bonis de Maripasoula et de Grand Santi devinrent français en 1965, par une demande collective d'inscription à l'état civil. Dans la mesure où ces populations ne possédaient ni acte de naissance, ni acte de baptême ou aucun autre document nécessaire pour établir leur nationalité, la régularisation se fit par un jugement déclaratif de naissance. Ce processus de francisation transforma alors des populations dites "indépendantes" en citoyens français, soumises sans distinction aux règles de l'état civil ainsi qu'aux droits et aux obligations liées à cette existence. Or, ces populations étaient souvent éloignées des enjeux liés à l'obtention de droits politiques. C'est ainsi que plusieurs ethnologues s'élevèrent pour dénoncer ce processus de francisation "forcée".

"Hurault précise dans plusieurs textes les réticences, sinon les refus, dont faisaient montre les Noirs marrons à l'égard de tout recensement pouvant permettre à l'État d'identifier des êtres dont l'histoire leur avait appris qu'il était préférable d'être ignoré des colonisateurs et des gouvernements. C'est ainsi que beaucoup d'entre eux ne déclarèrent pas tous les enfants d'une même famille, et cela au moins jusque dans les années 1970, par crainte de la conscription, ou simplement d'être identifié. " (Benoît, 2016 : 243)

Pour Françoise et Pierre Grenand, ces campagnes de recensement et de régularisation touchaient aux structures de parenté des populations. Par exemple, la transmission du nom de la mère en vertu des structures familiales matrilinéaires des Marrons et de certaines communautés amérindiennes constituait un système inversé aux règles familiales françaises patrilinéaires. D'autre part, pour les Amérindiens de l'intérieur, mais aussi pour les Bonis, il ne fallait pas attribuer un nom à un enfant avant qu'il ait donné des preuves de sa vigueur et de sa volonté de vivre. Lui donner un nom trop tôt l'exposerait à mourir (Grenand, Vernon, Li Chao, 1993 : 169). Pour André Cognat, autre détracteur de

11 Loi du 14 septembre 1951. 
ces campagnes, l'attribution de la nationalité française s'est faite au détriment du mode de vie transfrontalier des populations amérindiennes, les conduisant souvent à se sédentariser et à devoir choisir un État de référence. Des témoignages relatant l'attribution de noms et de prénoms donnent à penser que ces campagnes ont été organisées à la hâte et que les gendarmes n'étaient pas formés aux organisations sociales de ces peuples.

«Entre 1967 et 1969, environ 65 \% d'entre eux [Les Amérindiens] ont acquis la citoyenneté française, sans, semble-t-il, comprendre ce que cela signifiait, sauf, peut-être, pour ceux de la zone côtière. Leurs noms et leurs prénoms ont été francisés : un Amérindien n'a que son prénom, mais on sait d'où il sort. “Moi je n'ai que mon nom, mais ils l'ont francisé : imaginez qu'ils ont pris un calendrier et ont choisi un prénom : Guillaume. Ce sont vos parents qui l'ont choisi ? Même pas, c'est l'état civil. Pareil pour tous les Amérindiens".. (Propos d'Alexis Tiouka, k'alina de Awala-Yalimapo, in Géry et al., 2014 : 51)

Finalement, la question du recensement des personnes sans état civil ne fût pas réglée à la fin des années 1960 et la présence des services d'état civil dans les communes ne garantissait pas pour autant la déclaration et l'enregistrement des naissances. Benoît (2016:246) donne l'exemple de l'écart entre le nombre de naissances enregistré au centre de santé et celui à l'état civil. Dans les années 1990, ces écarts pouvaient aller jusqu'à quatre-vingt naissances par an. Entre 2004 et 2008, le nombre de naissances était tous les ans, supérieur à celui de l'état civil. D'autre part, l'enregistrement à l'état civil ne garantissait pas une identité administrative et juridique certaine. Des fautes d'orthographe ont pu être commises dans la rédaction des actes. Ces erreurs portant sur les noms, les prénoms des parents et des enfants, le sexe et le lieu de naissance, pouvaient être dues à un défaut d'attention des membres du dispensaire qui commettaient une erreur dans l'écriture de la déclaration (Doumeng, 2008 : 141), ou à une incompétence de ceux qui avaient la charge de l'état civil (Benoît, 2016 : 246). Le déclarant, dans certains cas non francophone ou analphabète, pouvait prononcer un nom phonétiquement et l'officier d'état civil le retranscrivait en méconnaissant l'orthographe. Entre le $1^{\text {er }}$ janvier et le 10 mars 2013, 1006 demandes de rectification en erreur matérielle sur les états civils, soit une moyenne de vingt-et-une demandes par jour, ont été déposées à la Maison de la Justice et du Droit de Saint-Laurent-du-Maroni (Benoît, 2016 : 248). Par ce nombre élevé de demandes, nous constatons que les erreurs sur les actes de naissance restent encore aujourd'hui très importantes. Ces dernières ont des conséquences sur le renouvellent de documents d'identité par exemple le passeport ou la carte d'identité, mais également dans l'obtention et le renouvellement d'un titre de séjour.

\section{De la catégorie de "primitif " à celle "d'étranger " : le cas des Marrons dans l'Ouest guyanais}

Dès les années 1980, il est possible de voir s'opérer un tournant dans le traitement des Marrons en Guyane, glissant de la catégorie de primitif à celle d'étranger. 
"Ces habitants passent progressivement, aux yeux des autorités, du statut de "primitifs" inoffensifs et locaux, à celui de migrants étrangers ou naturalisés, voire de réfugiés envahissants, illégitimes et indésirables. " (Léobal, 2015 : 477)

À travers l'étude d'un projet de déplacement des populations marronnes vers de nouveaux quartiers de relogement à Saint-Laurent-du-Maroni (Léobal, 2015 : 475-488), I'auteure montre comment les Marrons de Guyane sont peu à peu catégorisés par le prisme du droit des étrangers. Elle met en lumière l'amalgame établi entre les réfugiés et les Marrons installés de longue date dans la ville. Cette perception s'inscrit dans un contexte généralisé de lutte contre l'immigration illégale développée en Guyane et de contrôle de l'immigration engagée en France métropolitaine dès les années 1980.

"Si l'on accepte l'expression d'une "Europe forteresse" pour caractériser le développement de politiques migratoires européennes de plus en plus restrictives à l'égard de l'entrée et du séjour des étrangers des pays tiers, les départements français d'outre-mer constituent l'avant-poste d'une Europe forteresse qui ferme ses frontières non seulement sur ses limites sud et orientale, mais dès les territoires ultramarins français. "

(Benoît, 2015 : 464)

Plus localement, il est possible d'attribuer cette hostilité grandissante de la population et des élus locaux à l'arrivée massive de populations maronnes à Saint-Laurent-du-Maroni, fuyant la guerre civile du Suriname (1986-1992). Selon les sources (Bourgarel, 1989 ; Piantoni, 2009), au début du conflit, entre 9000 et 13000 personnes auraient fui le Suriname pour la Guyane. Elles s'établirent dans les villages frontaliers, dans la ville de Saint-Laurent-du-Maroni ${ }^{12}$ puis, pour celles sans famille sur place, dans des camps de réfugiés mis en place et gérés par l'armée française. L'État français et les élus locaux souhaitant voir partir ces populations, ils ne leur permirent pas d'obtenir le statut de réfugié. Qualifiées de "Personnes Provisoirement Déplacées du Suriname», elles n'avaient ni le droit de travailler, ni de scolariser leur enfant dans les écoles guyanaises. En décembre 1986 un poste-frontière est créé sur le fleuve Iracoubo, fermant ainsi l'est du territoire à la migration surinamienne. Cette situation se prolongea jusqu'en 1998. Rétabli peu de temps après, il existe encore aujourd'hui. Ce poste-frontière répond au dernier alinéa de l'article 78-2 du code de procédure pénale :

"Dans une zone comprise entre les frontières terrestres ou le littoral du département de la Guyane et une ligne tracée à cinq kilomètres de part et d'autre, ainsi que sur la route nationale 2 sur le territoire de la commune de Régina, l'identité de toute personne peut être contrôlée [...] en vue de vérifier le respect des obligations de détention, de port et de présentation des titres et documents prévus par la loi."

(Article 78-2, code de procédure pénale)

Au regard de cet article, deux postes de gendarmerie ont donc été installés sur la route nationale qui traverse la Guyane d'est en ouest, sur le pont de Régina et sur le fleuve Iracoubo. Selon I'article 4 de l'arrêté du barrage de Régina, la préfecture reconnaît "le caractère exceptionnel et dérogatoire au

12 Dans les années 1990, près d'un tiers des habitants de Saint-Laurent-du-Maroni était de nationalité surinamaise (Bechet, 2012 : 112). 
strict droit commun de ces contrôles permanents à l'intérieur du territoire [qui] doit être principalement ciblé sur la répression de l'orpaillage clandestin et de l'immigration clandestine" (Benoît, 2015 : 466). En effet, les politiques migratoires en Guyane sont marquées par toute une série de régimes dérogatoires au droit commun notamment en utilisant les articles 73 et 74 de la Constitution de 1958. Le droit commun est applicable dans les départements d'outre-mer, mais "le régime législatif et l'organisation administrative des départements d'outremer peuvent faire l'objet de mesures d'adaptation nécessitées par leur situation particulière" (article 73 de la Constitution). Ainsi, la Guyane française a recours à des dispositifs d'exception en matière de droit des étrangers, que cela soit dans la reconnaissance en paternité, les mesures de rétention et l'éloignement ou l'obtention de visas courts séjours pour les citoyens des pays régionaux.

\section{Maripasoula : une commune en marge de la société}

Créée en 1969 par le préfet Vignon, la commune de Maripasoula fête son cinquantième anniversaire. D'une superficie de plus de $18000 \mathrm{~km}^{2}$, elle est constituée d'un bourg qui regroupe la majorité de la population, ainsi que d'une multitude de villages implantés en bordure de rivière. Les principaux sont New Wacapou en aval du bourg et Aloïké et Tédamali-Boussoussa, tous deux en amont immédiat du bourg. Plus au sud de la commune, situés à plusieurs heures de navigation, se trouvent Elahé, Cayodé, Twenké, Taluen, Antecume Pata et Pidima constituant les villages du "pays indien" (rapport du plan local d'urbanisme, 2013 : 6). Dans ces villages, les services publics sont sommaires, se réduisant souvent à une école et un poste de santé. L'action publique sur le territoire est assez limitée notamment par des ressources financières plutôt faibles. Seul le bourg de la commune est pourvu d'institutions publiques : la mairie, la gendarmerie, l'armée de terre, le centre délocalisé de prévention et de soins, la protection maternelle et infantile et quelques associations. Nous recensons également dans le bourg, cinq établissements scolaires, dont un collège.

Si les populations marronnes et amérindiennes furent éloignées du droit français et de l'accès à un état civil, nous comprenons bien que ces dernières le furent aussi des institutions scolaires. Au moment de la colonisation, l'école ne concernait que les enfants de colons blancs. Après I'abolition de l'esclavage et l'instauration de la laïcisation en 1888, l'école intégra peu à peu les enfants d'esclaves libérés. Les populations amérindiennes et marronnes restèrent cependant à l'extérieur de ces changements, exclusion renforcée par le statut du territoire de I'Inini, ne soumettant pas à l'obligation scolaire leurs enfants. C'est à partir de la politique de "francisation" des années 1960, que commença la scolarisation des enfants de ces populations. La fin du régime de I'Inini en 1968, la dynamique démographique du département et la volonté d'assurer une éducation à l'ensemble des enfants ont permis la création d'écoles publiques dans les communes de l'intérieur de la Guyane. Ainsi, dans la commune de Maripasoula, l'école primaire du bourg a ouvert en 1968, suivie quatre ans plus tard par l'école de Twenké. Il a fallu attendre 1984 pour voir une école publique s'ouvrir à Antecume Pata et en 1998 à Pidima, dernier poste scolaire sur le fleuve 
Maroni. À la rentrée 2016-2017, environ $2000^{13}$ enfants étaient scolarisés dans la commune.

En dehors de l'école, il y a peu d'activités de loisirs. Les équipements sont limités et hormis des bâtiments religieux ${ }^{14}$ il n'existe pas de locaux spécifiquement dévolus aux activités culturelles. On retrouve seulement une bibliothèque implantée près de la mairie (rapport du plan local d'urbanisme, 2013 : 30). Cependant, la jeunesse est très présente sur ce territoire. Selon I'Insee ${ }^{15}$, en 2015, les zéro-quatorze ans représentaient $25,9 \%$ de la population. Si nous y ajoutons les quinze-vingt-neuf ans, nous atteignons $56,3 \%$ de la population. En l'espace de cinquante ans, la population communale a été multipliée par quinze, passant d'environ 700 habitants en 1969 à 12000 en 2018. Sa croissance démographique exponentielle est à la fois liée à un fort taux de natalité et à une immigration historique conséquente. À titre indicatif, en 2008, 3535 habitants étaient de nationalité française et 3021 étaient des personnes immigrées (rapport du plan local d'urbanisme, 2013 : 10). D'un point de vue sociocommunautaire, à l'instar de la Guyane, Maripasoula est multiculturelle et plurilingue. La population dominante, socialement et politiquement est la communauté Boni ${ }^{16}$, les populations amérindiennes, essentiellement Wayana et Teko sont moins importantes numériquement. Elles vivent principalement dans les villages de la commune. Nous retrouvons également des populations créoles guyanaises et antillaises ainsi que métropolitaines. Créoles et Métropolitains prétendent aux emplois publics les plus élevés : cadres de santé et personnels de l'éducation et de direction. En son bourg, nous recensons la présence de populations latinoaméricaines. Les populations brésiliennes et péruviennes s'inscrivent essentiellement dans le commerce et la restauration. Des populations caribéennes sont également présentes : dominicaines et haïtiennes.

Sur ce territoire, I'activité économique s'est développée lentement et principalement dans le secteur informel. Le taux de chômage avoisinait les 74,1 \% en 2014 contre 29,4 \% à Cayenne en 2015. Les activités économiques concernent aujourd'hui essentiellement le commerce de produits manufacturés, le transport fluvial de marchandises et de personnes, la vente des produits de la chasse, de la pêche, de l'agriculture, du bois et l'exploitation aurifère. Cette dernière est la seule activité industrielle sur le territoire (rapport du plan local d'urbanisme, 2013 : 14). Dès les années 1880, considérées comme la période de la ruée vers I'or, Maripasoula a vu s'installer sur ces berges des hommes originaires des Antilles anglaises pour travailler notamment sur les placers aurifères. L'activité minière connut un déclin avant d'être réinvestie par les populations Boni, puis dans les années 1990 cette fois-ci massivement de manière illégale, par des populations originaires du Brésil et du Suriname. Peu à peu, d'un profil d'hommes seuls, l'immigration s'est féminisée, au regard des évolutions migratoires

13 Chiffres 2016-2017. Pour le premier degré : affaires scolaires de Maripasoula, pour le second degré : rectorat de Guyane, constat d'effectifs d'élèves du second degré; Secteurs public et privé sous contrat en Guyane.

14 Lors des terrains d'enquête, neuf églises de confession différentes ont été recensées dans le bourg de la commune.

15 Sources : Insee, RP2015 (géographie au 01/01/2017) exploitations principales.

16 Parmi les populations marronnes on recense également des minorités Ndujka et Samaraka. 
mondiales. Ces familles vivaient entre le bourg de la commune et les placers aurifères. Cependant, les opérations successives de lutte contre l'orpaillage illégal menées par les militaires français ont conduit les populations migrantes en situation irrégulière à quitter le bourg de Maripasoula pour s'installer en face, sur les berges surinamiennes, peu occupées. Ainsi depuis les années 2000, de ce côté-là de la rive, des villages informels, des villes champignons se développent au gré des sites d'exploitation aurifère. Sur le bord du fleuve, juste en face de Maripasoula, se situe Antonio do Brigo ou Albina 2 où grands commerçants asiatiques vendent des objets de consommation courante, du matériel d'installation et d'orpaillage. En deuxième ligne, il y a un dédale de petites boutiques de vêtements et de parfums du Brésil, mais aussi des ongleries, des hôtels-restaurants, des bars et quelques habitations. Sur ce niveau-là de la rive surinamienne, il n'y a pas d'écoles, pas d'infrastructures ni d'institutions d'État. Une antenne du ministère de la Santé était cependant installée dans un petit préfabriqué. En 2018, sur les murs de ce bâtiment, il était possible de lire "se vende" et visiblement les agents du ministère avaient quitté les lieux depuis quelque temps. Sur cette rive, il n'y a ni accès à l'eau courante, ni à l'électricité. En aval, le long du fleuve, s'étend le village de Ronaldo. Ce village informel a été créé comme une base arrière de l'orpaillage illégal. II est constitué de populations brésiliennes, d'hommes, de femmes et d'enfants. En plus des habitations en bois qui se construisent et se vendent aussi vite, le village développe des services. Nous comptons la présence d'un garagiste, de restaurants, de commerces faisant la vente de produits en tout genre pour aller sur les sites d'orpaillage. Nous recensons également deux églises évangélistes, mais aucune école.

\section{Le droit à l'instruction : un parcours semé d'embûches}

\section{Une offre scolaire à deux vitesses}

Avec $50 \%$ de la population ayant moins de vingt-trois ans, la scolarisation est un enjeu majeur du territoire guyanais. Selon les chiffres du rectorat, à la rentrée 2016, 80659 élèves étaient inscrits pour environ 260000 habitants. En l'espace de dix ans, les effectifs ont progressé de 11,2\% dans le premier degré, de $18,2 \%$ au collège et de $50 \%$ au lycée (DEPP, 2017).Au côté du manque ${ }^{17}$ d'infrastructures scolaires manifeste sur le territoire, il est essentiel de prendre en compte son inégale répartition, au détriment des communes de l'Ouest guyanais, comme le souligne un rapport de la CNCDH publié au journal officiel en novembre 2017 :

"Une mobilisation d'envergure des pouvoirs publics est nécessaire pour rétablir l'équilibre, d'autant plus que la croissance démographique est principalement portée par les communes de l'Ouest guyanais, comme Grand-Santi, Maripasoula, Apatou et Mana, ainsi que Saint-Laurent-du-Maroni, cette dernière disposant toutefois de davantage d'infrastructures scolaires.» (CNCDH, 2017 : 16)

\footnotetext{
17 D'ici les dix prochaines années les besoins en constructions scolaires sont estimés à dix lycées, neuf collèges et 400 classes du premier degré, Données du ministère de I'Outre-mer, Pacte pour l'avenir de la Guyane (2016), in Yvette Mathieu, Accès aux droits et aux services publics en Guyane. Compte rendu du déplacement du Défenseur des droits en octobre 2016, p. 15.
} 
D'autre part, un rapport du Défenseur des droits sur l'accès aux droits et aux services publics en Guyane de 2016 met en lumière l'état des équipements et les conditions d'enseignement dans les écoles des fleuves :

"Beaucoup d'écoles dans les communes de l'intérieur sont dans un état de délabrement. Le problème récurrent est celui de l'entretien des locaux scolaires (dangerosité des locaux en raison de la présence de chauves-souris porteuses de maladies graves comme la rage et de la détérioration du bâti). Les internats sont pour la plupart dégradés.

Les écoles manquent de fournitures et de manuels scolaires. Moins d'un élève sur six bénéficie de la cantine. Le transport fluvial scolaire est insuffisant. Le nombre de places limitées par pirogue ne permet pas de prendre en charge tous les élèves ayant besoin de ce mode de transport. " (Mathieu, $2016: 16$ )

Sur ces territoires, le champ éducatif souffre également d'un déficit de professionnels formés. Le renouvellement important des équipes et le recours massif à un personnel contractuel ont une incidence sur la qualité de l'enseignement scolaire.

"Les écoles implantées le long des fleuves ne sont accessibles qu'en pirogue et les conditions de vie précaire dues à l'isolement, au climat et à l'habitat ne facilitent pas la tâche d'enseignants en lien avec une population en majorité non francophone. Le plus difficile est de stabiliser les enseignants titulaires, en raison de mauvaises conditions de vie et de logements inadaptés, voire inexistants. " (Mathieu, 2016 : 15)

Selon les chiffres de l'Insee, le taux de scolarisation ${ }^{18}$ des enfants âgés de six à quatorze ans avoisinait les $98 \%$ à Cayenne (2015) alors qu'il était de $92 \%$ à Maripasoula en 2014. Bien que toujours élevé, le véritable décalage se situe pour les jeunes âgés de quinze à dix-sept ans. Le taux de scolarisation de ce public était de $90 \%$ à Cayenne en 2015 contre 70,5 \% à Maripasoula en 2014. Ce taux bien plus faible s'explique notamment par l'absence de lycée dans la commune. En effet, les élèves des fleuves et de l'intérieur du territoire demeurent contraints de se rendre sur le littoral pour suivre leur scolarité au lycée et faire des études supérieures.

"La question de l'hébergement et de l'encadrement des jeunes collégiens ou lycéens contraints de quitter leur domicile pour poursuivre leur scolarité est cruciale. Les élèves peuvent être logés en internat ou en familles hébergeantes, chacune de ces modalités souffrant d'un manque de places et de nombreuses carences. [...] En Guyane, I'internat coûte cher, l'accueil n'est souvent pas prévu pendant les week-ends et les petites vacances scolaires et nombre d'entre eux ne proposent pas d'activités éducatives. Quant aux familles hébergeantes, elles sont souvent surchargées et auraient parfois tendance à se contenter d'offrir le gîte et le couvert. " (CNCDH, 2017 : 20)

Certains quittent leur environnement familial à l'entrée au collège vers l'âge de onze-treize ans. C'est notamment le cas des jeunes issus des villages du "pays indien", du Haut-Maroni. Ils sont accueillis prioritairement à l'internat du collège de Maripasoula. Pour la Commission nationale consultative des Droits de I’Homme, "concrètement, cela signifie un départ précoce de la famille,

18 Source : Insee. Pour Cayenne : RP2015 exploitation principale, géographie au 01/01/2017. Pour Maripasoula : Insee, RP2014 exploitation principale, géographie au $01 / 01 / 2016$ 
une perte de repères et une confrontation brutale avec la société guyanaise". Cependant, se déplacer pour poursuivre ses études induit de devoir passer le poste-frontière règlementant I'accès au littoral. Au-delà des résultats scolaires et des conditions socioéconomiques des familles, c'est la situation administrative et juridique du jeune et, par conséquent, la possession d'une identité papier qui constitue l'enjeu d'une possible scolarisation sur le littoral.

\section{Pratiques discriminatoires face à l'accès à l'école : la nécessité de produire des papiers}

Selon les directives ministérielles, relayées au plan académique et municipal, une inscription en école élémentaire se déroulerait en deux temps. Lors de la première inscription de l'enfant, il faut présenter à la mairie de rattachement, le livret de famille, la carte d'identité, une copie d'extrait d'acte de naissance de I'enfant ou tout autre document prouvant son identité et sa filiation. Les engagements internationaux ratifiés par la France et le préambule de la Constitution garantissent l'accès à l'école à tous les enfants. Tous ont le droit à l'éducation ${ }^{19}$, sur le principe d'égalité d'accès à l'instruction, à la formation professionnelle et à la culture ${ }^{20}$. Le ministère de l'Éducation nationale rappelle également, dans la circulaire $\mathrm{n}^{\circ} 002-063$ du 20/03/2002 ${ }^{21}$ que l'inscription dans un établissement scolaire d'un élève étranger ne peut être subordonnée à la présentation d'un titre de séjour. Malgré ces dispositions légales, plusieurs collectifs et des fédérations syndicales font état des pratiques discriminatoires face à la scolarisation des enfants étrangers et des fleuves.

Dans certaines mairies de Guyane, des documents supplémentaires seraient parfois demandés, par exemple le titre de séjour des parents. Ainsi, dans un rapport intermédiaire de 2016, le Défenseur des droits se doit de rappeler aux communes une délibération prise le 7 mai 2013 : "l'inscription à l'école se fait sur présentation de justificatifs d'identité et d'un certificat de vaccination DT polio. L'exigence de tout autre document aboutit à une différence de traitement qui pourrait revêtir un caractère discriminatoire" (Matthieu, 2016 : 17). Un an plus tard, la $\mathrm{CNCDH}$, à son tour :

"[Elle] déplore vivement la persistance de pratiques extrêmement hétérogènes d'une commune à l'autre au moment de la procédure d'inscription dans une école maternelle ou élémentaire. Malgré les rappels successifs adressés aux maires quant aux dispositions légales en vigueur, la situation ne semble pas s'être améliorée de façon notable.»

(CNDH, $2017: 38$ )

Les rappels de ces organismes mettent en lumière un manquement constant aux directives officielles. Dans sa délibération $n^{\circ}$ 2009-318 du 14/09/2009, la Halde $^{22}$ relevait que nombre d'enfants en Guyane n'étaient pas déclarés à la

19 Article 2 du protocole additionnel de la Convention européenne de sauvegarde des droits de l'homme et des libertés fondamentales du 18 mai 1952.

20 Préambule de la Constitution du 27 octobre 1946, intégré dans la Constitution de 1958.

21 La circulaire $n^{\circ} 2002-063$ du 20/03/2002 relative aux modalités d'inscription et de scolarisation des élèves de nationalité étrangère du premier et second degré réaffirme "qu'aucune distinction ne peut être faite entre les élèves de nationalité française et de nationalité étrangère, pour l'accès au service public d'éducation ".

22 HALDE : Haute Autorité de Lutte contre les Discriminations et pour l'Égalité. 
naissance. Pour pallier cette difficulté, l'Observatoire de la non-scolarisation du rectorat de Guyane préconisait d'établir l'identité de l'enfant au moyen d'une attestation de notoriété publique. En dépit de ces recommandations, il semblerait que les mairies continuent d'exiger un acte d'état civil comme condition subordonnant l'inscription scolaire.

"Selon les travailleurs sociaux de la maison des adolescents de Saint-Laurent-duMaroni, les dossiers d'inscription scolaire, dépourvus d'actes d'état civil ou de justificatif de domicile, sont refusés ou accusent un retard si considérable dans leur traitement qu'il en résulte bien souvent une déscolarisation. " (LDH, $2014: 48$ )

En contradiction avec I'article L. 131-5 du code de l'éducation, certaines mairies requièrent également une facture d'eau, d'électricité ou bien un bail alors que nombre de familles vivent en habitat autoconstruit ${ }^{23}$. Cette dérive entraînerait des stratégies de contournement, pour se procurer une adresse fictive, soit auprès d'un proche, soit auprès d'un tiers moyennant parfois une contrepartie financière.

"À Cayenne, la problématique relative à la constitution du dossier d'inscription scolaire tient à la production d'un justificatif de domicile. II est en effet exigé un justificatif de moins de trois mois, la liste des justificatifs acceptés étant limitée : société guyanaise des eaux ou EDF ou France Télécom ou la taxe d'habitation. " (LDH, 2014 : 48)

Ainsi, selon une association installée depuis plus d'une vingtaine d'années dans un quartier d'immigration de la commune de Remire-Montjoly ${ }^{24}$ il faudrait attendre près d'un an pour qu'un enfant migrant trouve une place dans une école élémentaire. On constate un phénomène similaire dans l'ouest du territoire. L'antenne de I'Unicef a remarqué que l'effectif global des écoles maternelles et élémentaires était bien inférieur à leur capacité d'accueil globale à la rentrée 2016. Or, au sein des ateliers jeunesse de l'Unicef, plusieurs enfants de nationalité étrangère, non scolarisés, allophones et âgés de six à neuf ans, sont placés sur liste d'attente. D'autre part, la fermeture du CASNAV ${ }^{25}$ de Cayenne à la rentrée 2016 puis son changement d'adresse à la rentrée suivante ont contribué à renforcer l'exclusion des enfants étrangers devant s'inscrire dans le second degré. À la rentrée 2016, les services académiques ont enregistré plus de 800 nouvelles familles avec la nécessité de scolariser près 400 enfants.

Une fois passée la première inscription en mairie, pour les élèves du premier degré, les familles doivent se rapprocher de l'école de rattachement. Elles procéderont ainsi à un nouveau dépôt de documents, conservés dans les dossiers scolaires des enfants, sous forme de dossiers papier ou numériques. Qu'ils soient originaires des fleuves ou étrangers, les enfants doivent traverser un labyrinthe administratif dans lequel le papier, qu'il soit un acte de naissance ou un justificatif de domicile devient un véritable "sésame". Cependant, une fois l'enfant inscrit, les papiers semblent s'effacer voire même devenir invisibles, inintéressants aux yeux des acteurs qui les ont pourtant demandés, et exigés.

23 Les maisons de fortune représentaient à Maripasoula en 2014, 31,1 \% des résidences principales contre $2,9 \%$ à Cayenne un an plus tard. Source : Insee, op. cit.

24 Commune limitrophe à celle de Cayenne.

25 CASNAV : Centre Académique pour la Scolarisation des élèves allophones Nouvellement Arrivés et des enfants issus de familles itinérantes et de Voyage. 


\section{Mettre en mots des écrits pour reconstruire des parcours}

\section{Une impression de vide dans les dossiers scolaires}

Pour cet article, il a été choisi d'étudier deux types de papier, au sein du collège de Maripasoula. Nous nous sommes intéressées à seize dossiers d'élèves inscrits en cinquième et quatrième inclus en UPE2A en 2016 et aux vingt-trois fiches numériques d'inscription pour les élèves inclus en UPE2A, toutes classes confondues, en 2017. La consultation des dossiers papier a eu lieu en janvier 2017 dans la salle des professeurs du collège. Pendant la pause méridienne, la salle était vide, seules quelques feuilles volaient au vent sur la grande table centrale. C'est en ouvrant ces dossiers que me sont revenues en tête les paroles des acteurs sollicités pour les consulter. Que cela soit l'équipe de direction ou des professeurs, ils étaient unanimes : "I/ n'y a rien à voir dans les dossiers". En effet, la première impression à la consultation des seize dossiers a été celle d'un vide, d'une absence, d'un silence autour de l'existence des enfants et des adolescents. Les nombreuses ratures, l'écriture enfantine ainsi que le peu d'informations écrites invitaient à penser que les élèves avaient pour la plupart complété eux-mêmes les dossiers. Les parents n'écrivant pas ou peu ${ }^{26}$, I'enfant écrit ce qu'il sait, ce qu'il connaît, mais aussi peut-être, ce qu'il peut dire. Que les dossiers soient vides ou manquants, ils marquent par l'absence, la présence de ces enfants et adolescents dans l'établissement. Sur les traces écrites, I'existence administrative de l'enfant se résume à quelques éléments de base parfois incomplets : le nom et le prénom de l'enfant, éventuellement celui de son représentant légal. Certains documents comportent le nom des enfants à charge, mais toutes les dates de naissance ne sont pas connues.

"L'un des dossiers attira notre attention. Il s'agissait du dossier de Benjamin, un jeune garçon affecté en classe de quatrième à la rentrée 2016. Dans une écriture infantile, nous lisons son nom, son prénom, sa date de naissance et son lieu de naissance : le Suriname.

Dans la partie "Responsables de l'élève", aucun nom n'est indiqué, seulement la case

"mère seule" est cochée. II n'y a aucune adresse du représentant légal de l'élève. II y

a un numéro de téléphone vraisemblablement surinamien, au regard de l'indicatif, mais ce dernier est illisible. Nous savons par la case "mère seule" cochée une seconde

fois qu'elle est la personne à contacter en cas d'urgence. Dans la case "profession du responsable", il est écrit "sans". Sur la dernière page du dossier, nous constatons que les signatures de l'élève et du représentant légal se ressemblent. " (Notes de terrain, 2018)

Cette impression de vide se retrouve dans les fiches numériques consultées en 2018. Cette fois-ci, nous avons ressenti l'absence autour des familles des jeunes, que cela soit celle du père ou de la fratrie. Sur les vingt-trois fiches, dix-huit mentionnaient un seul représentant légal dont quatorze seraient des mères. Sur les vingt-six représentants légaux, vingt se déclaraient sans emploi. "Sans" père, "sans" emploi, toujours ce qualificatif "sans", marqueur de l'absence. Ce petit aperçu reflète la société paupérisée de Maripasoula avec une atrophie du commerce formel et une place prépondérante de l'informalité.

26 Pour rappel, $39 \%$ de la population est en situation de difficulté avec l'écrit et une personne sur cinq est en situation d'illettrisme sur le littoral de Guyane, donnés du ministère de l'outre-mer, op. cit. 
Toujours selon ces fiches d'inscription, dix-sept élèves seraient nés à l'étranger. Cependant, seulement huit ont eu une scolarité en N-1 à l'étranger. Parmi ces huit élèves, deux sont nés en Guyane française. Ces éléments interrogent le public inscrit en UPE2A. D'ordinaire et selon la circulaire de cadrage, dans ce dispositif sont accueillis de jeunes allophones nouvellement arrivés sur le territoire dans les six derniers mois. Or, en Guyane, dans les dispositifs UPE2A, nous pouvons retrouver des jeunes nés et scolarisés sur le territoire ou des jeunes arrivés depuis plusieurs années. Cette commune s'inscrit dans un contexte frontalier, où les populations sont mobiles. Qu'elles soient françaises ou étrangères, elles sont pour la plupart non francophones ou allophones.

"À en croire la fiche d'inscription de Benjamin, il ne serait pas arrivé dans l'établissement en 2016 en classe de quatrième' mais en 2015 en classe de cinquième. Ici, commence une confrontation d'informations pour reconstruire le parcours de cet enfant. Selon la fiche d'évaluation du professeur UPE2A, cet élève serait "allophone et arrivé en mars 2017". En 2016 et en 2017, il était inscrit en UPE2A, dispositif destiné aux primo-arri-

vants. " (Notes de terrain, 2018)

Contrairement à l'impression de vide, de silence laissé par l'écriture des dossiers, les documents justificatifs sont très nombreux, voire même abondants en fonction des élèves. Sur dix pièce ${ }^{27}$ à fournir, quatre sont systématiquement présentes : le relevé d'identité bancaire, la pièce d'identité de l'enfant ou son acte de naissance, un justificatif de domicile et une attestation scolaire. Sur seize dossiers consultés pour l'année 2016-2017, tous les élèves avaient au moins deux adresses en fonction des justificatifs. Nous avons recensé chez certains élèves jusqu'à cinq adresses différentes dans la commune de Maripasoula. D'autre part, les seize dossiers étaient constitués d'une attestation d'hébergement chez un tiers. Ce tiers garant de l'hébergement des jeunes voire de leur famille semble, au regard des papiers, être né dans le pays d'origine des élèves : Haïti, République dominicaine, Pérou, Brésil, Suriname.

\section{Des mobilités et des déplacements passés sous silence}

Selon son dossier scolaire, Lucas est un jeune garçon né en 2007 au Suriname. II a un passeport brésilien. Selon la fiche d'inscription de la professeure d'UPE2A, il est arrivé dans l'école en avril 2016 et a été inscrit en classe de CE2. Lucas est né à Paramaribo en 2007 où ils ont vécu, lui et sa mère Paola, avant de rejoindre Cayenne. La mère de Lucas est brésilienne, ce qui explique le passeport de son fils. Son père aurait été tué lors d'un règlement de compte sur un site d'orpaillage alors que Lucas n'était qu'un bébé. En 2013, un homme aurait reconnu l'enfant. Martiniquais comme le précise Paola, elle pense que Lucas pourrait devenir français. Parmi les dispositifs particuliers liés aux droits des étrangers, dans la loi du 24 juillet 2006, le collectif outremer met en avant les procédures renforcées de contrôle des "paternités de complaisance" (collectif

27 Dans le dossier d'inscription, il est demandé : trois photographies récentes, la notification d'affectation, le relevé d'identité bancaire, la photocopie de la pièce d'identité de I'élève ou un extrait d'acte de naissance, un justificatif de domicile ou une attestation d'hébergement, une photocopie du livret de famille, une attestation d'assurance scolaire, la photocopie des pages vaccinations du carnet de santé de l'élève, une contribution facultative concernant I'adhésion au foyer socio-éducatif de cinq euros, une contribution facultative à I'association sportive de cinq euros. 
outre-mer, 2007 : 1). Sur ce point, la lecture de l'article 108 de la loi du 24 juillet 2006 dispose que "lorsqu'il existe des indices sérieux laissant présumer que la reconnaissance d'un enfant est frauduleuse, l'officier de l'état civil saisit le procureur de la République", lequel peut s'opposer à la reconnaissance. La question de la reconnaissance en paternité, médiatisée ${ }^{28}$ en Guyane, constitue un enjeu de taille dans la régularisation des familles. Bien que punissables par la loi, de nombreuses familles espèrent obtenir, par ce moyen, la nationalité française pour leurs enfants. Or, ces familles se retrouvent souvent isolées du droit pour avoir voulu le contourner et sans recours juridique. Cependant, Paola n'a pas fait les papiers, car elle a quitté Cayenne cette même année pour retourner à Paramaribo avec Lucas. Ils y resteront trois ans avant d'arriver à Maripasoula en avril 2016.

En 2016-2017, dans son dossier, une adresse indique qu'il vit dans le bourg de Maripasoula avec sa mère. II est alors en classe de CM1 et inscrit en UPE2A. Contrairement à I'adresse indiquée dans son dossier, Lucas explique timidement qu'il vit dans le village de Ronaldo situé de l'autre côté de "l'eau ". Le fait de résider de l'autre côté du fleuve est vécu comme un secret par bon nombre d'enfants et de jeunes, qu'il convient de cacher plus spécifiquement à certaines catégories d'acteurs. De leur côté, les professionnels de l'éducation restent très évasifs, voire même silencieux. Tout le monde sait que des enfants vivent de l'autre côté du fleuve, mais personne ne semble vouloir en parler. Seul le principal adjoint du collège s'est exprimé clairement sur le sujet. Pour lui, de nombreux élèves vivraient sur la rive surinamienne en face de Maripasoula, mais auraient une boite postale du côté français. Lors d'un échange avec des acteurs associatifs, nous apprenons qu'une domiciliation chez un particulier coûterait 400 euros dans le bourg de Maripasoula. À I'heure actuelle, aucune association ne propose de domiciliation. Ces propos sont à mettre en corrélation avec le rapport du Défenseur des droits de 2016 :

"L'accès à l'information s'opère, comme sur un marché, selon un prix, celui du déplacement, de la traduction, de l'écrivain public, du ticket de passage pour une place favorable dans la file d'attente. Le recours à des intermédiaires communautaires, illégaux et payants, génère des trafics en tout genre (fraude documentaire, vol de courriers, malversation, racket). " (Mathieu, $2016: 33$ )

Ainsi, à son arrivée, Paola a obtenu une boite postale à Maripasoula et a installé son fils dans une petite maison en bois, au cœur du village de Ronaldo. La maison, sans eau courante ni électricité, entourée de commerces, comporte deux pièces : une cuisine et une chambre. II y a une seule fenêtre, située dans la cuisine. Dans la chambre, il y a un hamac installé au-dessus du lit. Paola dort dans le lit et Lucas dans le hamac au-dessus de sa mère.

En octobre 2016, à travers un dessin réalisé en classe, Lucas nous explique qu'il vit avec "son papi, sa mamie et son chat à Ronaldo». À son arrivée à Ronaldo, Lucas est très vite confié à un tiers. Sa mère doit se rendre à l'hôpital de Saint-Laurent-du-Maroni pour accoucher. En effet, à Maripasoula les femmes enceintes sont rapatriées à Cayenne puisque le centre délocalisé de prévention

28 Maro Laurent (2015) Procès en appel des faux papas chinois, Guyane Première, 05/02/2015. 
et de soins ne permet pas de faire des accouchements. Mais lorsque la patiente n'est pas en mesure de produire une carte de séjour ou une pièce d'identité française, les professionnels de santé doivent saisir l'Agence Régionale de Santé et la préfecture pour obtenir un laissez-passer. Sans titre de séjour valide, Paola a préféré partir en pirogue ${ }^{29}$ à Saint-Laurent-du-Maroni et elle est revenue par le même chemin avec le nouveau-né. En janvier 2017, nous apprenons par Lucas qu'il est de nouveau confié à un tiers, une amie de sa mère. Paola est partie en forêt, sur un site d'orpaillage avec sa petite sœur.

À la rentrée 2017, Lucas est absent. II est inscrit en décembre lors de son arrivée dans l'école. II fait son année en classe de CM2 ainsi qu'en UPE2A. Pour cette année, dans son dossier scolaire, une nouvelle adresse à Maripasoula est donnée. Entre septembre et décembre 2017, Lucas a suivi sa mère sur un site d'orpaillage. C'est Paola qui nous le dit, Lucas lui affirme qu'il était à Paramaribo. Selon elle, elle préférait avoir ses enfants près d'elle plutôt que de les laisser à Ronaldo. Pour elle, ils n'étaient pas sécurité. Au moment de cette discussion, en janvier 2018, nous sommes dans la nouvelle maison de Lucas. La maison se situe dans le bourg de Maripasoula et comprend deux pièces : une cuisine et une chambre. II y a une grande fenêtre dans la chambre qui donne sur un jardinet et une autre dans la cuisine au niveau de l'évier. Comme nous l'a dit Paola, c'est le même prix qu'à Ronaldo, mais là il y a l'eau et l'électricité. Elle paye 250 euros par mois. Elle nous dit que le village de Ronaldo n'est pas bon pour les enfants. Cependant, très peu de temps après, en avril, Lucas est de nouveau retourné vivre à Ronaldo. Paola a finalement acheté une maison. Cette maison de trois pièces et deux terrasses, toute en bois, sans eau ni électricité a coûté 263 grammes d'or. Elle se situe au début du village de Ronaldo et donne directement sur le fleuve. Elle est bien plus grande que les deux précédentes et toujours arrangée avec beaucoup soin. Paola travaillant dans I'orpaillage illégal, cette maison lui permet de maintenir son activité - notamment pour stocker des denrées alimentaires à envoyer sur les camps - tout en donnant à ses enfants un espace pour vivre. Cependant, les enfants ne sortent pas seuls au village. Quand Lucas souhaite faire du vélo, sa mère appelle une pirogue et paye cinq euros pour aller à Maripasoula. C'est d'ailleurs le tarif quotidien pour que Lucas aille à l'école.

Ainsi en presque deux ans, Paola, Lucas et sa petite sœur auront déménagé trois fois entre le bourg de Maripasoula et le village de Ronaldo. Lucas aura été confié à un tiers, puis emmené en site d'orpaillage, impactant ainsi sa scolarité. Cependant, ces informations restent absentes de son dossier scolaire et de la connaissance des professeurs de l'école. Le cas de Lucas n'est pas isolé à Maripasoula, où comme nous l'avons vu, I'orpaillage prend une place importante dans l'économie. Entre 2015 et 2016, une fratrie de trois enfants d'origine brésilienne, installée à Ronaldo, a été inscrite à l'école primaire. Selon les registres d'inscription, I'un des trois enfants est arrivé à Maripasoula quelques mois avant ses deux sœurs. En avril 2017, la fratrie a été radiée de l'école. Deux enfants sont partis avec leur mère et leur grand-mère à Paramaribo, l'ainée est restée à Ronaldo chez sa tante. En âge d'entrer au collège, elle est finalement déscolarisée depuis plus d'un an. Aujourd'hui, elle est au Brésil.

29 II faut compter au minimum cinq heures de pirogue en saison des pluies et deux jours en saison sèche. 


\section{Des fratries gommées sur le papier}

Lara et Jessica ont été inscrites au collège de Maripasoula à la rentrée scolaire 2016. Lara était inscrite en quatrième et Jessica en cinquième. Les deux sœurs ont un an d'écart et sont toutes les deux nées en République dominicaine. Aucune autre trace de leur scolarité antérieure n'est présente dans le dossier. Les deux sœurs sont arrivées en avril 2016 à Maripasoula. Elles ont toutes deux été très surprises par la réalité de ce territoire. Elles s'attendaient à arriver dans une ville moderne. L'année où nous nous sommes rencontrées, entre 2016 et 2017, les deux jeunes filles étaient assidues en classe. Elles ont toutes les deux passé le DELF $A 1^{30}$ qu'elles ont obtenu. Lara voulait étudier à Cayenne pour devenir architecte. Jessica, elle voulait devenir styliste. Dans un atelier collectif organisé en janvier 2017, auquel ont participé les jeunes filles, les adolescents étaient invités à imaginer l'histoire d'une famille migrante. Pour les jeunes filles en présence, l'école semblait être un levier pour poursuivre la migration vers Cayenne ou vers Paris et ainsi obtenir des "papiers".

Elles ont la même adresse, à Maripasoula avec une attestation d'hébergement, une facture EDF et la copie de la carte nationale d'identité de la personne domiciliant la famille. Elles vivent avec leur mère, selon leur dossier scolaire. Or, sur l'attestation d'hébergement des deux adolescentes, il est mentionné le nom de leur mère et celui $d^{\prime}$ 'un homme. En effet, les jeunes filles vivent avec leur mère et leur beau-père. Ce dernier tient un garage à domicile et a racheté une boite de nuit dans le bourg. La maison est en bois. Elle est au milieu d'un terrain qui doit servir d'entrepôt pour le garage. Sur le terrain, il y a deux ou trois quads, une moto et un $4 X 4$. Face au chemin, il y a deux fenêtres très souvent fermées. De I'autre côté, il y a une terrasse. La table en plastique et trois chaises installées sur cette terrasse sont entourées d'un amoncèlement d'objets obsolètes, de bouteilles de bière en verre et d'outils de mécanicien. Un groupe électrogène est relié à l'intérieur de la maison. La maison ne semble donc pas être raccordée au réseau électrique. Nous avons évoqué les conditions de vie au village de Ronaldo. Néanmoins, du côté français, les familles font face à une grande précarité de l'habitat. Selon I'Insee ${ }^{31}$, en 2014, 37,8 \% des résidences principales étaient déclarées avoir une douche ou une baignoire et des W.-C. à l'intérieur du logement, contre $91,9 \%$ à Cayenne en 2015. Seulement 3,2 \% des résidences étaient reliés au tout à l'égout contre 53,1 \% à Cayenne en 2015.

Dans le dossier de Laura, on trouve les documents d'une troisième jeune fille Clara et dans sa fiche d'inscription de 2017, il est indiqué que la mère a trois enfants à charge. Dans le dossier de Jessica, deux sœurs sont indiquées : Lara et Clara. Clara n'a pas le même nom de famille que les deux autres. Toujours dans le dossier de Lara, nous découvrons le nom d'une autre personne à contacter, celle de l'homme présent sur l'attestation d'hébergement. Il a le même nom de famille que Clara. Le beau-père des jeunes filles a lui-même une fille, Clara. Elle est arrivée la même année que les sœurs. À la rentrée 2017, Clara est partie à Cayenne pour poursuivre ses études au lycée. Elle est maintenant majeure et "sans papier», mais poursuit sa scolarité. Elle vit chez un membre de la famille.

30 DELF A1 : Diplôme d'études en langue française, niveau A1 du Cadre européen commun de référence pour les langues.

31 Source : Insee, op. cit. 
À la rentrée 2017, Laura est inscrite en troisième et en UPE2A, Jessica elle est en quatrième seulement. Lara et Jessica sont toujours à Maripasoula. La mère des jeunes et le beau-père sont souvent absents. Ainsi leur mère a fait venir une amie qui vit aussi dans la maison pour qu'elle garde les deux jeunes filles. En 2017-2018, leur rapport à l'école a changé. Elles sont très absentes. Lara n'a presque pas mis les pieds au collège depuis janvier 2018 selon ses professeurs. Elle n'a d'ailleurs pas passé son diplôme national du brevet en fin d'année. Fuyant l'école, nous retrouvons Laura dans la boite de nuit de son beau-père où elle travaillerait, ou encore sur la place des fêtes tard le soir en compagnie d'amis. En un an, Lara a maigri. En mars 2018, elle a été transportée à Cayenne après avoir tenté de se suicider. C'est à ce moment-là qu'arrive dans la maison, Alex, le frère de Clara. II est le deuxième enfant du beau-père. Alex arrive de République dominicaine où il a laissé sa mère avec qui il a toujours vécu. En classe, il parle peu et devient nostalgique quand il parle de sa mère. II a quatorze ans et apprend à composer avec cette nouvelle famille qu'il ne connaissait pas. À son arrivée, il est inscrit en classe de quatrième et en UPE2A. II suit douze heures en UPE2A par semaine jusqu'à la fin de l'année. Pour l'orientation, il a été demandé le redoublement de Lara en classe de troisième. Jessica et Alex, eux, passeront en classe de troisième. Alex finit l'année avec l'une des meilleures moyennes de sa classe.

Par les traces papier de cette famille, nous constatons une absence de données sur la migration des enfants. Aucun document ne matérialise leur vie et par exemple leur scolarité en République dominicaine. Seul le passeport peut faire acte d'une migration ou d'un déplacement. De plus, I'arrivée tardive des enfants non francophones ne semble pas être un élément pris en compte dans leur parcours scolaire. Alex, arrivé en mars 2018, passe en classe de troisième après trois mois de scolarité en France. Selon la professeure UPE2A, il ne parlait pourtant pas français en fin d'année. La migration, en plus d'être absente des dossiers, ne semble donc pas être un élément à prendre en compte pour comprendre l'expérience scolaire des enfants et des adolescents.

\section{Conclusion}

Grâce à l'étude des dossiers scolaires et des traces écrites, nous avons souhaité mettre en lumière les parcours de mobilité et de migration des enfants vivant sur le fleuve Maroni. Nous avons proposé d'ancrer notre réflexion dans le territoire de Maripasoula, commune frontalière du Brésil et du Suriname en Guyane française. À travers cet article, nous avons mis en évidence un système paradoxal autour de la question des papiers, qu'ils soient acte de naissance, justificatif d'hébergement ou dossiers scolaires. D'une part, le papier est apparu comme une nécessité pour accéder aux droits et notamment pour s'inscrire à l'école. Il est le sésame de l'inscription légitime, offrant également une existence administrative sur le territoire. D'autre part, nous avons constaté qu'une fois exigés et collectés, les papiers n'intéressaient plus les acteurs qui les avaient pourtant demandés, comme tombés dans I'oubli. Selon Dardy (1998, 37), I'accomplissement d'actes administratifs, d'actes d'inscription ne serait qu'un rite $d^{\prime}$ insertion sociale d'une société d'écritures. 
À travers un apport sociohistorique sur l'accès aux droits - droit à une existence et droit à l'instruction - dans les territoires de l'Ouest guyanais, nous avons mis en exergue le manque de reconnaissance des populations, tant dans I'histoire collective des communautés que dans les trajectoires individuelles. Pour étudier ces trajectoires individuelles, nous nous sommes intéressées aux dossiers scolaires des enfants et des adolescents présents dans un dispositif de l'Éducation nationale, I'UPE2A. Nous avons constaté de nombreux silences, des absences et des ruptures. Des silences se sont manifestés autour des antécédents scolaires et des parcours de migration ou de mobilité. L'absence s'est ressentie quand il s'agissait d'évoquer les liens de parenté et les membres de la fratrie. Enfin, des ruptures apparaissaient autour des lieux de vie et de la scolarité. Qu'ils soient "élève allophone nouvellement arrivé " ou "élève allophone non nouvellement arrivé", ces enfants sont invisibilisés dans leur expérience scolaire. Le premier l'est tant la question de l'allophonie est généralisée sur ce territoire et le second, car considéré comme Français, il ne peut pas être migrant. Parce que né sur le territoire, l'enfant n'effectuerait pas de déplacement.

Cette mise sous silence des trajectoires individuelles interroge enfin la place donnée à I'histoire collective de l'immigration. Cette idée renvoie à un article de Blickstein (2011 : 103) qui met en avant l'oubli des migrations régionales dans I'histoire de l'immigration en Argentine : "Cette immigration rendue silencieuse a enfanté des traces vivantes, qui se manifestent par les diverses formes d'exclusion et d'invisibilité que subissent les "migrants de la frontière" dans leur vie quotidienne". Dès lors, nous pourrions interroger la place donnée aux migrations régionales et aux mobilités dans la construction de l'histoire guyanaise, société postcoloniale, à l'instar de l'Argentine.

\section{Références bibliographiques}

Bassargette Denis et Di Meo, Guy (2008) Les limites du modèle communal français en Guyane : le cas de Maripasoula. Les Cahiers d'Outre-Mer, 61 (241242), pp. 49-80.

Béliard Aude et Biland Émilie (2008) Enquêter à partir de dossiers personnels, Genèses, 70, pp. 106-119.

Benoît Catherine (2016) Pampila et politique sur le Maroni : de l'état civil sur un fleuve frontière, Histoire de la justice, 26, pp. 237-59.

Benoît Catherine (2015) Sans-Papiers amérindiens et noirs marrons. La fabrication de l'étranger sur le fleuve Maroni, in Jean Moomou Éd., Les marronnages et leurs productions sociales, culturelles dans les Guyanes et le bassin caribéen du XVIlème au XXème siècle : bilans et perspectives de recherche, Cayenne, Ibis Rouge, pp. 463-472.

Blickstein Tamar (2011) L'oubli au cœur de la mémoire, Hommes et migrations. Revue française de référence sur les dynamiques migratoires, 1293, pp. 96-106.

Bourgarel Sophie (1989) Migration sur le Maroni : les réfugiés surinamais en Guyane, Revue Européenne des Migrations Internationales, 5 (2), pp. 145-153. 
Cayouette-Remblière Joanie (2011) Reconstituer une cohorte d'élèves à partir de dossiers scolaires. La construction d'une statistique ethnographique, Genèses, 85, pp. $115-133$.

Commission nationale consultative des droits de l'homme (2017) Avis sur l'effectivité du droit à l'éducation dans les outremers. Regard particulier sur la Guyane et Mayotte, Paris, $96 \mathrm{p}$.

Dardy Claudine (1998) Identités de papiers, Paris/Montréal, L'Harmattan, 198 p.

Dardy Claudine (1997) L'identité-papier, Les cahiers de médiologie, 4, pp. 225-231.

Doumeng Valérie (2008) Un contenu inadapté : I'acte de naișsance des populations marginales de la Guyane française, in Claire Neirinck Éd., L'état civil dans tous ses états, Paris, Droit et société, L.G.D.J, pp. 133-147.

Insee (2018) Dossier complet commune de Cayenne, [en ligne] consulté le 10/05/2018. URL : https://www.insee.fr/fr/statistiques/2011101?geo=COM-97302

Insee (2018) Dossier complet commune de Maripasoula, [en ligne] consulté le 10/05/2018. URL : https://www.insee.fr/fr/statistiques/2011101?geo=COM-97353

Insee (2018) Dossier complet commune de Saint-Laurent-du-Maroni, [en ligne] consulté le 10/05/2018. URL : https://www.insee.fr/fr/statistiques/2011101?geo $=$ COM -97311

Farge Arlette (2003) Le bracelet de parchemin. L'écriture sur soi au XVIIle siècle, Paris, Bayard, 113 p.

Géry Yves, Alexandra Mathieu et Christophe Gruner (2014) Les abandonnés de la République : vie et mort des Amérindiens de Guyane française, Paris, Albin Michel, $352 \mathrm{p}$.

Grenand Françoise, Vernon Diane et M. Li Chao (1993) Personnes et familles, in Jean-Pascal Martres et Jacques Larrieu Éds., Coutumes et droit en Guyane: Amérindiens, Noirs-Marrons, Hmong : actes du colloque de Cayenne 25, 26 et 27 juin 1992, Paris, Economica, 217 p.

Jamoulle Pascale (2013) par-delà les silences : non-dits et ruptures dans les parcours d'immigration, Paris, La Découverte, $280 \mathrm{p}$.

Jolivet Marie-José et Diane Vernon (2007) Droits, polygamie et rapports de genre en Guyane, Cahiers d'études africaines, 47 (187-188), pp. 733-752.

Léobal Clémence (2015) Bidonvilles et camps de réfugiés : le tournant des années 1980 pour la catégorisation des marrons à Saint-Laurent-du-Maroni, in Jean Moomou Éd., Les marronnages et leurs productions sociales, culturelles dans les Guyanes et le bassin caribéen du XVIlème au XXème siècle : bilans et perspectives de recherche, Cayenne, Ibis Rouge, pp. 475-490.

Ligue des Droits de I'Homme (2014) La carence institutionnelle dans l'établissement de l'état civil, vecteur d'atteintes à l'accès aux droits dans l'Ouest guyanais, Rapport de mission du groupe de travail "outre-mer», Paris, $44 \mathrm{p}$.

Mairie de Maripasoula (2013) Rapport de présentation du plan local d'urbanisme, commune de Maripasoula, Guyane française, $131 \mathrm{p}$.

Mathieu Yvette (2016) Accès aux droits et aux services publics en Guyane. Compte rendu du déplacement du Défenseur des droits en octobre 2016, Défenseur des droits, Paris, $50 \mathrm{p}$. 
Noiriel Gérard (1993) L'identification des citoyens. Naissance de l'état civil républicain, Genèses, 13 (1), pp. 3-28.

Piantoni Frédéric (2009) L'enjeu migratoire en Guyane française : une géographie politique. Espace outre-mer, Matoury, Guyane, Ibis Rouge Éditions, 448 p.

Thabouillot Gérard (2015) Amérindiens et Noirs-Marrons au temps de I'Infini : la contribution politique des tribus de frontière à la construction de la Guyane française contemporaine (1930-1969), in Jean Moomou Éd., Les marronnages et leurs productions sociales, culturelles dans les Guyanes et le bassin caribéen du XVIlème au XXème siècle : bilans et perspectives de recherche, Cayenne, Ibis Rouge, pp. 437-450.

Urban Yerri (2015) Les Marrons et le droit en Guyane française de 1836 à la francisation, in Jean Moomou Éd., Les marronnages et leurs productions sociales, culturelles dans les Guyanes et le bassin caribéen du XVIlème au XXème siècle: bilans et perspectives de recherche, Cayenne, Ibis Rouge, pp. 427-436. 


\section{Alexandra Vié \\ École et migration en Guyane française : invisibilité des parcours migratoires dans les dossiers scolaires à Maripasoula}

Dans cet article, nous proposons d'interroger la migration par le prisme de I'école en Guyane française. Puisque l'accès à la scolarité est conditionné par la production de documents administratifs, nous avons choisi de travailler à partir de dossiers scolaires d'enfants et d'adolescents. L'absence d'écriture dans les dossiers, la récurrence des attestations d'hébergement chez un tiers, la pluralité des adresses sont autant d'éléments qui mettent en avant la manière dont les enfants et leurs familles pratiquent le territoire et par conséquent l'école. En confrontant différents récits, nous tenterons de dégager des pistes de réflexion sur la mise en mots des parcours migratoires des enfants et des jeunes migrants, à Maripasoula, ville d'Amazonie, située à la frontière du Brésil et du Suriname.

\section{School and Migration in French Guiana: Invisibility of Migratory Routes in the Scholastic Folders in Maripasoula}

In this article, we propose to question the migration by the prism of the school in French Guiana. The access to the education is conditioned by the administrative papers. We have therefore chosen working from the scholastic folders of the children and the adolescents. Illiteracy can be seen with the absence of writing. The attestations of lodging mention frequently several addresses. All of this information explain how the children and their families practice our territory and the school. By the confrontation of this different narration and experiment, we will try to expose some words about the migration experience of the children and the young migrants in Maripasoula, Amazonia town, placed between the Brazil and the Suriname.

\section{Escuela y migración en la Guayana francesa: invisibilidad de las rutas migratorias en los registros escolares en Maripasoula}

En este artículo, proponemos cuestionar la migración por el prisma de la escuela en la Guayana francesa. Como el acceso a la educación está condicionado por los documentos, fue elegido trabajar a partir de los registros escolares de niños y adolescentes. La falta de escritura, la repetición de los certificados de residencia, la pluralidad de las direcciones son elementos que resaltan la forma en que los niños y las familias practican el territorio y por lo tanto la escuela. Al confrontar diferentes historias, trataremos de encontrar algunas ideas para poner en palabras las rutas migratorias de niños y jóvenes migrantes en Maripasoula, ciudad de la Amazonia, en la frontera de Brasil y de Surinam. 